\title{
Determination of local energy density functionals from Brueckner-Hartree-Fock calculations
}

\author{
D. Gambacurta, ${ }^{1}$ L. Li ${ }^{2}$ G. Colò, ${ }^{3}$ U. Lombardo, ${ }^{1}$ N. Van Giai, ${ }^{4}$ and W. Zuo ${ }^{5}$ \\ ${ }^{1}$ Dipartimento di Fisica and INFN-LNS, Via S. Sofia 64, I-95123 Catania, Italy \\ ${ }^{2}$ State Key Lab Nuclear Physics and Technology, School of Physics, Peking University, Beijing 100871, China \\ ${ }^{3}$ Dipartimento di Fisica, Università degli Studi, and INFN Sezione di Milano, via Celoria 16, I-20133 Milano, Italy \\ ${ }^{4}$ Institut de Physique Nucléaire, IN2P3-CNRS and Université Paris-Sud, F-91405 Orsay, France \\ ${ }^{5}$ Institute of Modern Physics, Chinese Academy of Sciences, Lanzhou 730000, China
}

(Received 3 May 2011; published 8 August 2011)

\begin{abstract}
Skyrme-like energy density functionals are built upon new ab initio calculations of nuclear matter which reproduce the empirical saturation properties. These calculations are performed in the framework of the Brueckner-Hartree-Fock approximation with consistent two- and three-body forces. The Skyrme parametrizations are obtained from a simultaneous fit of the spin-isospin components of the potential energy of symmetric nuclear matter and of the experimental energies and charge radii of a number of closed-shell and closed-subshell nuclei. These parametrizations are tested on the energies and charge radii of the $\mathrm{Sn}$ isotopic chain and on the ${ }^{208} \mathrm{~Pb}$ giant resonances, showing a substantial improvement of the LNS functional obtained in a previous fit. The comparison with standard Skyrme forces can give some hints on how to reconcile nuclear matter and finite nuclei.
\end{abstract}

DOI: 10.1103/PhysRevC.84.024301

PACS number(s): 21.65.Mn, 21.30.-x

\section{INTRODUCTION}

The experimental data already accumulated and still expected from exotic beams challenge the theoretical description of the nuclear structure based on Hartree-Fock (HF) calculations with energy density functionals (EDF). The determination of an EDF easy to use, and at the same time capable of predicting nuclear properties in extended regions of the nuclear chart is a longstanding goal. Among the various choices the Skyrme-type parametrizations belong to the most widely used functional because of their simple local structure. As for the predictive power this is more difficult to assess because the phenomenological parametrizations are by essence adjusted in the regions of known nuclei, and the calculated nuclear properties may extrapolate diversely when going away from these regions. This is the motivation for attempting to build parametrizations which incorporate theoretical guidelines such as the equation of state (EoS) of neutron matter from variational calculations [1] in the case of the SLyn model [2], or the Brueckner-HF (BHF) EoS of nuclear matter [3] in further Syrme-type parametrizations [4,5].

In Ref. [5] BHF calculations of the nuclear-matter energy and effective mass were fitted with a Skyrme-type EDF, and a new Skyrme parametrization, named LNS, was obtained. The effective mass was involved in the fit because it is associated with finite-range corrections included in the Skyrme interaction. The LNS force was tested by means of HF calculations of closed-shell and closed-subshell nuclei. The results for the binding energies and charge radii were considered quite promising, if one takes into account that there were no parameters adjusted on nuclei except the spin-orbit parameter $W_{0}$. However, LNS was not able to reproduce nuclear properties with a quality comparable to other Skyrme forces. It seems that within the EDF approach (not restricted to the Skyrme-type ansatz: see, for instance Ref. [6]), in addition to the bulk component taken from BHF calculations, it is necessary to introduce a purely phenomenological finite-range component to get a good description of nuclear properties.

In this paper the limits of a Skyrme parametrization based on BHF $a b$ initio calculations are investigated and overcome by performing a simultaneous fit of nuclear matter and finite nuclei. The new fitting procedure is not applied to the total potential energy per particle $U$, but to its components $U_{T S}$ in spin-isospin space. The reason is that $U$ results from a strong cancellation of the four components $U_{T S}$. This also amounts to constrain independent observables, such as spin and isospin symmetry energies. From such a choice of the fit one can expect a strong reduction of the class of Skyrme parametrizations that reproduce the nuclear-matter EDF.

We take advantage of the new BHF calculations [7] performed with a nucleon-nucleon interaction in which twoand three-body forces are consistently treated, in the framework of a meson-exchange model. The main result is a better description of the saturation properties of symmetric and asymmetric nuclear matter. In particular, the empirical saturation point is pretty well reproduced.

The paper is organized as follows. In Sec. II the BHF theory with consistent two- and three-body interactions is reviewed and the main results on the saturation properties of nuclear matter are reported. Section III is devoted to describe the fitting procedure, and the new Skyrme parametrizations are discussed. The latter are tested on the ground-state and pairing properties of the $\mathrm{Sn}$ isotopic chain, and on selected giant resonance energies obtained using fully self-consistent HF plus random phase approximation (RPA) calculations, in Sec. IV. Concluding remarks are drawn in Sec. V.

\section{EQUATION OF STATE OF NUCLEAR MATTER FROM BHF CALCULATIONS}

The Brueckner theory with three-body forces reproduces the empirical saturation properties of nuclear matter. 
But the Brueckner predictions extend far below and above the saturation density; therefore, one can try to build up an EDF from $a b$ initio Brueckner calculations.

\section{A. Brief review of the Brueckner theory}

The Brueckner theory with two- and three-body forces is described elsewhere $[3,7,8]$. Here we simply give a brief review of the BHF approximation which is the starting point of the present calculations. The main quantity is the reaction $G$ matrix, which satisfies the Brueckner-Bethe-Goldstone (BBG) equation:

$$
G(\omega)=v_{N N}+v_{N N} \sum_{k_{1} k_{2}} \frac{\left|k_{1} k_{2}\right\rangle Q_{k_{1}, k_{2}}\left\langle k_{1} k_{2}\right|}{\omega-\epsilon_{k_{1}}-\epsilon_{k_{2}}} G(\omega),
$$

where $k_{i} \equiv\left(\vec{k}_{i}, \sigma_{i}, \tau_{i}\right)$ denotes the single-particle (s.p.) momentum, the spin and isospin variables, respectively, and $\omega$ is the starting energy. The $G$ matrix, the Pauli operator $Q$ and the s.p. energies $\epsilon_{k}=k^{2} / 2 m+U_{k}$ depend separately on the neutron and proton densities. The auxiliary potential $U_{k}$ is chosen as

$$
U_{k}=\sum_{k^{\prime}}\left\langle k, k^{\prime}\left|G\left(\varepsilon_{k}+\varepsilon_{k^{\prime}}\right)\right| k, k^{\prime}\right\rangle_{A} \theta\left(k_{F}-k^{\prime}\right),
$$

where the subscript denotes antisymmetrization. The interaction $v_{N N}$ is

$$
v_{N N}=V_{2}^{\text {bare }}+V_{3}^{\text {eff }},
$$

where $V_{2}^{\text {bare }}$ is the bare nucleon-nucleon force and $V_{3}^{\text {eff }}$ is an effective two-body force obtained from the three-body force $W_{3}$ by averaging on the third particle according to

$$
\begin{aligned}
\left\langle\vec{r}_{1} \vec{r}_{2}\left|V_{3}^{\text {eff }}\right| \vec{r}_{1}^{\prime} \vec{r}_{2}^{\prime}\right\rangle= & \frac{1}{4} \operatorname{Tr} \sum_{n} \int \mathrm{d} \vec{r}_{3} \mathrm{~d} \vec{r}_{3}^{\prime} \phi_{n}^{*}\left(\vec{r}_{3}^{\prime}\right) \psi\left(r_{13}^{\prime}\right) \psi\left(r_{23}^{\prime}\right) \\
& \times W_{3}\left(\vec{r}_{1}^{\prime} \vec{r}_{2}^{\prime} \vec{r}_{3}^{\prime} \mid \vec{r}_{1} \vec{r}_{2} \vec{r}_{3}\right) \phi_{n}\left(r_{3}\right) \psi\left(r_{13}\right) \psi\left(r_{23}\right) .
\end{aligned}
$$

In this equation $\psi(r)=1-\eta(r)$ is the correlated two body wave function and $\eta(r)$ the defect function. The latter is determined from the $G$ matrix according to

$$
\eta_{12}=\left\langle\vec{r}\left|\frac{Q}{H_{0}-\epsilon_{k_{1}}-\epsilon_{k_{2}}} G\right| k_{1}, k_{2}\right\rangle,
$$

so that $V_{3}^{\text {eff }}$ must be calculated self-consistently with the $G$ matrix and the s.p. potential $U_{k}$ on the basis of the BBG iterative procedure. It is clear from Eq. (4) that the effective force arising from the $3 \mathrm{BF}$ in nuclear medium is density dependent via the defect function. A detailed description and justification of the method can be found in Refs. [8,9].

The three-body force used in nuclear matter calculations is based on a meson-exchange model, including virtual particlehole excitations of the vacuum (relativistic corrections) and the low-energy virtual excitations of nucleons, $\Delta(1232)$ and $N^{*}$ (1440) [9]. The corresponding diagrams are shown in Fig. 1. Adopting a two-body force described in terms of meson exchange the same meson parameters (masses, coupling constants, and cutoffs) can also be used in the three-body force. This makes the whole interaction fully consistent and realistic,
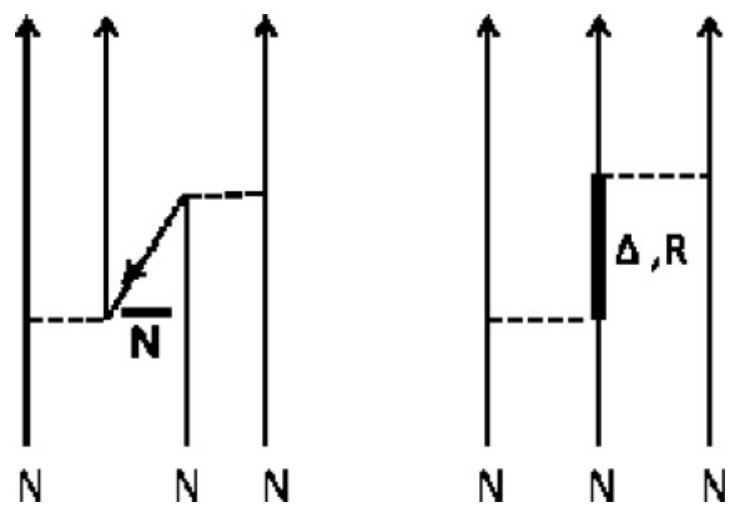

FIG. 1. Three-body force diagrams included in the BHF calculations. On the left: $N \bar{N}$ virtual excitations. On the right: $\Delta$ and Roper resonance excitations (see text).

because the meson parameters are fixed so as to reproduce the experimental phase shifts of the nucleon-nucleon scattering. At this point one should observe that the use of phenomenological three-body forces together with realistic two-body forces [6] somehow breaks the $a b$ initio nature of the Brueckner approach.

Recently, we have adopted for the two- and three-body forces the parameters of the Bonn-B potential $V_{2}^{\text {bare }}$ [10]. Details and results using this interaction are presented in Refs. [7,11]. The main merit of this choice is that it improves the agreement with the empirical saturation properties of nuclear matter, including the values of symmetry energy and compression modulus.

From the EoS of symmetric nuclear matter the saturation density is estimated to be about $\rho_{0} \simeq(0.16-0.17) \mathrm{fm}^{-3}$, with a corresponding energy $E / A=-16 \mathrm{MeV}$ and the compression modulus $K_{\infty}=220 \mathrm{MeV}$. From the EoS of isospin-asymmetric matter one can extract the density dependent symmetry energy. Its value at the saturation point is about $30 \mathrm{MeV}$. Along with with the energy per particle, the effective mass ( $k$ mass), self-consistently calculated in $\mathrm{BHF}$ approximation, is to be considered. As recalled in the Introduction, it is associated with the nonlocality correction to the energy functional. In the density domain $0.11 \leqslant \rho \leqslant 0.2$ $\mathrm{fm}^{-3}$ its range is $0.60 \leqslant m^{*} / m \leqslant 0.69$.

\section{B. Nuclear matter potential energy in spin and isospin subspaces}

In the homogeneous nuclear medium the spin $S$ and isospin $T$ of the interacting pairs of nucleons are conserved quantum numbers (the Coulomb interaction does not appear), and the energy per particle can be decomposed into a kinetic part plus the $T S$ components of the interaction part:

$$
\frac{E}{A}=\frac{K}{A}+\sum_{T S} U_{T S} \text {. }
$$

The individual contributions $U_{T S}$ have a direct relation with global nuclear matter properties. It is well known that, in a broad range of densities, the energy per particle of isospinasymmetric nuclear matter (isospin-ANM) is quadratic in the 

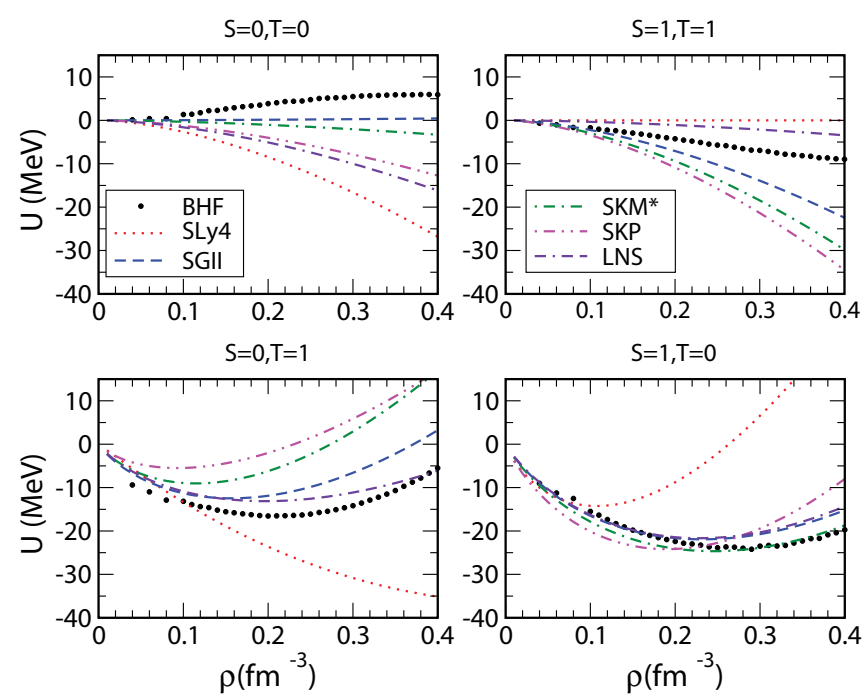

FIG. 2. (Color online) Spin-isospin components of the BHF potential energy in comparison with a few standard Skyrme forces: SLy4 [2], SGII [15], SKM* [14], SKP [16], and LNS [5].

isospin asymmetry parameter $\beta=(N-Z) / A$ [12]. Similarly, the energy per particle of spin-ANM is quadratic in the spinasymmetry parameter $\sigma=\left(A_{\uparrow}-A_{\downarrow}\right) / A[13]$ :

$$
\begin{aligned}
& U(\rho, \beta)=U(\rho)+\beta^{2} a_{s}(\rho), \\
& U(\rho, \sigma)=U(\rho)+\sigma^{2} a_{\sigma}(\rho) .
\end{aligned}
$$

The two functions $a_{s}(\rho)$ and $a_{\sigma}(\rho)$ govern most properties of isospin- and spin-polarized nuclear matter, respectively. Since both parabolic laws are valid in the whole range of spin and isospin values, it follows that

$$
\begin{aligned}
& a_{s}(\rho)=U(\rho, \beta=1)-U(\rho, \beta=0)=-U_{00}-U_{10}, \\
& a_{\sigma}(\rho)=U(\rho, \sigma=1)-U(\rho, \sigma=0)=-U_{00}-U_{01} .
\end{aligned}
$$

In our previous construction of Skyrme-type energy functionals from $a b$ initio calculations leading to the LNS force the overall potential energy of symmetric nuclear matter was adjusted, and the spin and isospin ANM energies were put as constraints [5]. Adjusting simultaneously the individual $T S$ components seems to be more adequate for the sake of restricting the class of the equivalent parametrizations, a desirable ultimate goal of such investigation. The four $T S$ components of the potential energy per particle calculated by the BHF approach with two-body and three-body forces are shown in Fig. 2.

In the same figure, those components the potential energy calculated with various Skyrme forces are shown for comparison. Despite the quite noticeable differences in various channels between the Skyrme and BHF results, strong compensations give rise to similar behaviors of the symmetric matter EoS, at least in the saturation region, as seen in Fig. 3.

\section{FITTING PROCEDURE}

The basic microscopic inputs in our Skyrme fit are the BHF isospin-spin potential energy components $U_{T S}^{(\mathrm{BHF})}$, discussed in

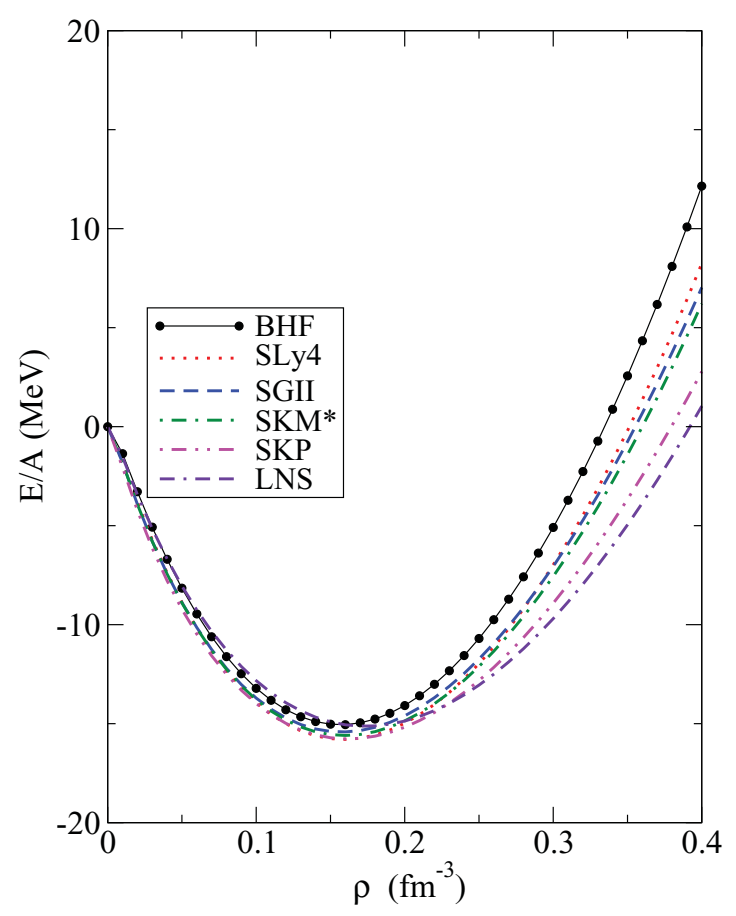

FIG. 3. (Color online) BHF vs. Skyrme energy per particle in symmetric nuclear matter.

the previous section. In the Appendix the standard analytic expressions of $U_{T S}^{\text {Skyrme }}$ calculated with Skyrme-type interactions are presented.

Before we discuss the procedure for determining the optimal values of the Skyrme parameters, some comments are in order. From Fig. 2 we can see how typical Skyrme interactions compare with the BHF approach. Despite of giving a quite similar and good reproduction of the saturation properties (as shown in Fig. 3), the BHF and Skyrme-HF approaches sizeably deviate, especially with increasing density, when looking at the separate $T S$ channels.

Figure 2 shows that in the $(T, S)=(0,0)$ channel $U_{00}^{(\mathrm{BHF})}$ is small and positive with a slight negative concavity. By looking at Eq. (A2), we see that $U_{00}^{\text {(Skyrme) }}$ depends only on the $\left(t_{2}, x_{2}\right)$ Skyrme parameters. In order to reproduce the sign of $U_{00}^{(\mathrm{BHF})}$ the quantity $t_{2}\left(1-x_{2}\right)$ has to be positive. On the other hand, since the analytic form of $U_{00}^{\text {(Skyrme) }}$ implies that the second derivative of the function has the same sign as the function itself, it is impossible to reproduce at the same time the sign and the concavity of $U_{00}^{(\mathrm{BHF})}$. This means that the analytical structure of the Skyrme force makes it quite hard to fit the $U_{T S}^{(\mathrm{BHF})}$ channels separately, at least as far as the $U_{00}^{(\mathrm{BHF})}$ is concerned. A similar comment can be made for $U_{11}^{(\mathrm{BHF})}$ as well.

The fitting procedure adopted in this paper consists in minimizing the quantity

$$
\begin{aligned}
\chi^{2}= & \sum_{i=1}^{N_{p}}\left(\frac{U_{T S}^{(\mathrm{BHF})}(i)-U_{T S}^{(\text {Skyrme })}(i, t, x)}{\delta_{U}(i)}\right)^{2} \\
& +\sum_{i=1}^{N}\left(\frac{E^{(\mathrm{Exp})}(i)-E^{(\mathrm{SHF})}(i, t, x)}{\delta_{E}(i)}\right)^{2}
\end{aligned}
$$




$$
\begin{aligned}
& +\sum_{i=1}^{N}\left(\frac{r_{c}^{(\mathrm{Exp})}(i)-r_{c}^{(\mathrm{SHF})}(i, t, x)}{\delta_{r}(i)}\right)^{2} \\
& +\left(\left.P(x, t)\right|_{\rho=\rho_{0}} / \delta_{P}\right)^{2} .
\end{aligned}
$$

Here, $E^{(\mathrm{Exp})}\left(r_{c}^{(\mathrm{Exp})}\right)$ and $E^{(\mathrm{SHF})}\left(r_{c}^{(\mathrm{SHF})}\right)$ are, respectively, the experimental and Skyrme HF (SHF) total binding energies (charge radii) of the doubly closed shell nuclei included in the fit, namely ${ }^{16} \mathrm{O},{ }^{40} \mathrm{Ca},{ }^{48} \mathrm{Ca},{ }^{56} \mathrm{Ni},{ }^{78} \mathrm{Ni},{ }^{100} \mathrm{Sn},{ }^{132} \mathrm{Sn}$, and ${ }^{208} \mathrm{~Pb}$. The number of fitted nuclei is $N=8$ while $N_{p}=40$ since we fit ten values of energy per particle, for ten different values of the density in the density range $0<\rho \leqslant 2 \rho_{0} \mathrm{fm}^{-3}$ in the four $T S$ channels. The last term in Eq. (11) indicates the constraint on the pressure, $P=0$, at the saturation density $\rho_{0}=0.16 \mathrm{fm}^{-3}$. With the simplified notation $(t, x)$ we denote the set of Skyrme parameters $\left(t_{i}\right.$ and $x_{i}$ with $\left.i=0, \ldots, 3\right)$. The spin-orbit parameter $W_{0}$ is determined so as to reproduce the splitting of the $p_{\frac{1}{2}}-p_{\frac{3}{2}}$ states in ${ }^{16} \mathrm{O}$. The exponent $\sigma$ in the density-dependent term is kept fixed at the value $\sigma=\frac{1}{6}$. Some attempts have been done to fit Skyrme forces with the same protocol and keeping $\sigma=\frac{1}{3}$, without any significant improvement.

In our procedure we also impose the compression modulus $K_{\infty}$ and the symmetry energy $a_{s}$ to take values between 220-245 MeV and 28-32 MeV, respectively. This was done by imposing in the $\chi^{2}$ minimization two linear constraints, corresponding to $K_{\infty}$ and $a_{s}$, of the type

$$
F_{m} \leqslant F(x, t) \leqslant F_{M},
$$

where $F(x, t)$ is a linear combination of the $t$ and $x$ parameters [see Eqs. (A6) and (A7)]. No constraint is imposed to the effective mass.

The weights $\delta_{U}$ and $\delta_{E}$ in Eq. (11) have a double meaning. From one side, they can be interpreted as errors (standard deviations) of the data points. On the other side, by suitably tuning them, one can increase or reduce the weight of each point, or each subset of points, in the $\chi^{2}$ minimization. In particular, reducing the values of $\delta_{E}(i)$ and $\delta_{r}(i)$ one can put more weight on the phenomenological constraints (i.e., the experimental binding energies and charge radii) with respect to the microscopic (BHF) ones.

In the fitting procedure, we use for the BHF data different values of $\delta_{U}$ in different density regions. For all $T S$ channels the values of $\delta_{U}(i)$ are expressed as percentages of the corresponding $U_{T S}$ values. As already seen in Fig. 2, the deviations of the Skyrme isospin-spin components from the BHF results, at least for the considered interactions, are quite large especially at higher density. Therefore, in order to obtain a reasonable reproduction of the BHF results also at higher density (i.e., up to $2 \rho_{0}$ ) we choose smaller $\delta_{U}(i)$ values when increasing the density. More precisely we use $\delta_{U}(i)=10 \%$ for $\rho<0.1 \mathrm{fm}^{-3}, \delta_{U}(i)=5 \%$ for $0.1 \mathrm{fm}^{-3} \leqslant$ $\rho \leqslant 2.5 \mathrm{fm}^{-3}$ and $\delta_{U}(i)=1 \%$ for $2.5 \mathrm{fm}^{-3}<\rho \leqslant 2 \rho_{0}$. This choice is also motivated by the worse numerical stability of our BHF calculations at lower densities, i.e., $\rho \leqslant 0.08 \mathrm{fm}^{-3}$, where it is more difficult to reach a good degree of convergence in the BHF iterative procedure. Thus, we prefer to use larger values of $\delta_{U}(i)$ for $\rho<0.1 \mathrm{fm}^{-3}$.
In order to get reasonably small deviations from the experimental binding energies of nuclei, small values of $\delta_{E}(i)$ have to be adopted. For all nuclei we take the same $\delta_{E}$, ranging from $\delta_{E}=0.5 \mathrm{MeV}$ (LNS5) to $\delta_{E}=0.1 \mathrm{MeV}$ (LNS1). In the following we only show the results for these two extreme cases.

Figure 4 shows the errors on the experimental total binding energies and charge radii corresponding to different values of $\delta_{E}$. As expected, a better agreement is actually found when reducing $\delta_{E}$. For the binding energies, we note that the overall performance of the force is deteriorated in the case of light nuclei and it is much better if we exclude them. Otherwise, looking at Fig. 5 (note that different scales have been used with respect to Fig. 2), we can see that a less satisfactory fit of the BHF isospin-spin potential energy components is found, when $\delta_{E}$ gets smaller. This is also seen in Table I, where the root mean square (rms) deviations for the total binding energies $\left(\sigma_{E}\right)$, charge radii $\left(\sigma_{r}\right)$, and potential energies $U_{T S}$ in the different $T S$ channels $\left(\sigma_{U}\right)$ are shown for the two sets. However, in the LNS1 case the agreement with the BHF isospin-spin components is still better than those shown in Fig. 2 using other Skyrme sets. Moreover, in Fig. 6 we see that the total energy per particle is not strongly affected by the different values of $\delta_{E}$ used in the fit.

In Table II we report the values of the parameters $t_{i}, x_{i}$, and $W_{0}$, corresponding to LNS5 and LNS1, while in Table III the corresponding main bulk nuclear matter properties are given. For comparison, in the same table, we also show the results obtained with the LNS and SLy4 sets and the estimated BHF values.

In Table IV we compare the experimental and the calculated total binding energies and charge radii. One can see that the charge radii do not change very much when reducing $\delta_{E}$, while a quite different behavior is found for the binding energies. In particular, we see that with LNS5 the deviations are quite large, of the order of $10 \mathrm{MeV}$ for almost all the considered nuclei, but they become much smaller with LNS1, the largest deviation being $5.95 \mathrm{MeV}$ for ${ }^{40} \mathrm{Ca}$.

We add some more comments on the details of Fig. 4, in which the relative energy deviations (upper panel) and charge

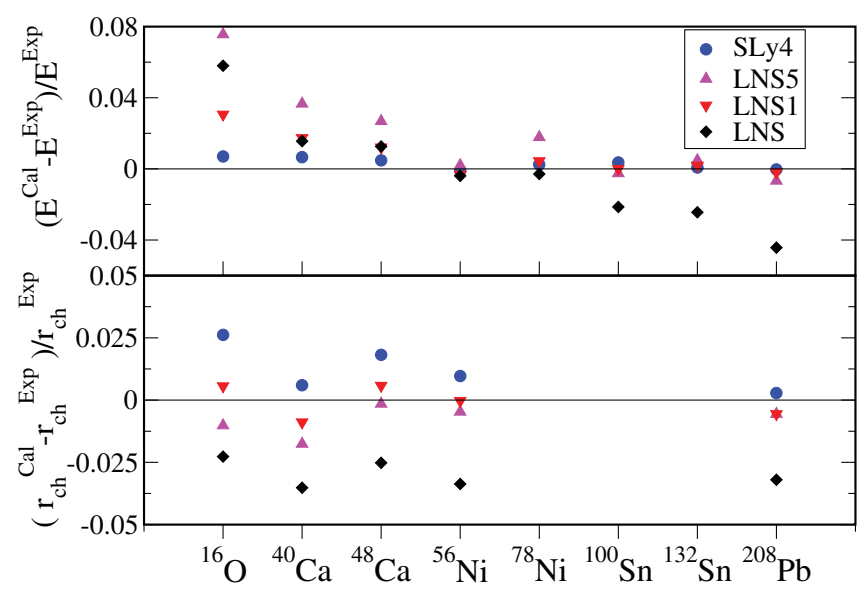

FIG. 4. (Color online) Energy deviations (upper panel) and charge radii deviations (lower panel), calculated with LNS5 and LNS1, are compared with the results of SLy4 and LNS. 

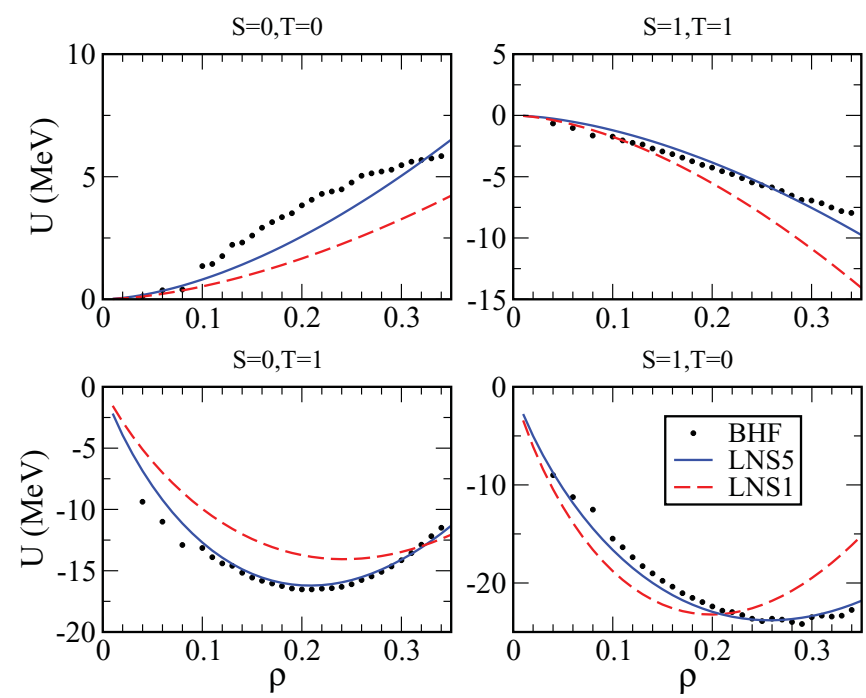

FIG. 5. (Color online) Spin-isospin components of the potential energy from the BHF calculations compared with the results from LNS5 and LNS1.

radii deviations (lower panel) are shown. Comparing the LNS and LNS5 results, we see that the latter are in better agreement with the experimental energies only heavy nuclei $\left({ }^{100} \mathrm{Sn},{ }^{132} \mathrm{Sn}\right.$ and ${ }^{208} \mathrm{~Pb}$ ), while a worse behavior is found for the others. On the contrary, the LNS1 set gives results systematically better than LNS except for the two Ca isotopes where the agreement is nearly the same. For medium-heavy nuclei, i.e., from ${ }^{56} \mathrm{Ni}$ up to ${ }^{208} \mathrm{~Pb}$, we see that the binding energies obtained for LNS 1 are of the same quality as the SLy4 ones, while the latter are better for ${ }^{16} \mathrm{O}$ and for the two $\mathrm{Ca}$ isotopes. As far as the charge radii are concerned we see that both results corresponding to LNS1 and LNS5 are much better than the LNS ones and in many cases also better than the SLy4.

In conclusion, as mentioned above, we find that the new results obtained with LNS1 and LNS5 present a more natural trend than those obtained with LNS, namely they are better for medium-heavy nuclei and worse for light nuclei, as it can be expected if an $a b$ initio calculation of infinite matter plays a key role in the fit.

\section{APPLICATIONS}

\section{A. The Sn isotopic chain}

The Skyrme parametrizations obtained in the last section can be tested on nuclei not used in the fitting procedure. Here we specifically select the chain of Sn even isotopes. A complete treatment of these nuclei would require a HF-Bogoliubov

TABLE I. Root mean square deviations of total binding energies $\left(\sigma_{E}\right)$, charge radii $\left(\sigma_{r}\right)$ of nuclei, and $U_{T S}$ potential energies $\left(\sigma_{U}\right)$, calculated with LNS5 and LNS1.

\begin{tabular}{lccc}
\hline \hline & $\sigma_{E}[\mathrm{MeV}]$ & $\sigma_{r}[\mathrm{fm}]$ & $\sigma_{U}[\mathrm{MeV}]$ \\
\hline LNS5 & 9.08 & 0.0342 & 0.86 \\
LNS1 & 3.54 & 0.0226 & 2.53 \\
\hline \hline
\end{tabular}

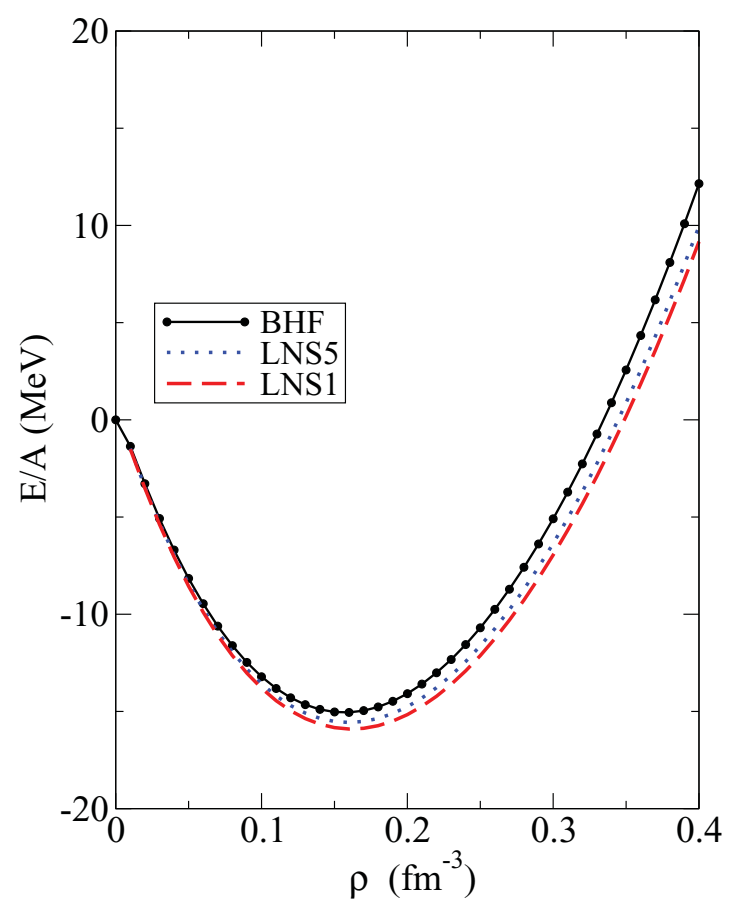

FIG. 6. (Color online) Energy per particle for symmetry nuclear matter: BHF vs. Skyrme parametrizations of Table II.

(HFB) calculation. But, since our aim here is just to see whether the present interactions can reasonably compare with other Skyrme forces as far as the overall trend with $N-Z$ is concerned, we limit ourselves to the HF-Bardeen-CooperSchrieffer (HF-BCS) framework. We adopt the pairing force

$$
V=V_{0}\left(1-\frac{\rho\left(\vec{r}_{1}+\vec{r}_{2}\right)}{\rho_{0}}\right) \delta\left(\vec{r}_{1}-\vec{r}_{2}\right) .
$$

The HF-BCS equations are solved setting the systems in a large box with a radius $R=20 \mathrm{fm}$. We employ a radial mesh of $0.1 \mathrm{fm}$, and the BCS equations are solved, as usual, within a pairing window: only the levels up to the $N=126$ neutron shell are considered. Among the unbound levels we keep only those corresponding to single-particle resonances. They are determined increasing the box radius up to $25 \mathrm{fm}$, and

TABLE II. LNS5 and LNS1 Skyrme parameters obtained in the fits. The exponent $\sigma$ (last line) is fixed.

\begin{tabular}{lrr}
\hline \hline & \multicolumn{1}{c}{ LNS5 } & \multicolumn{1}{c}{ LNS1 } \\
\hline$t_{0}\left(\mathrm{MeV} \mathrm{fm}^{3}\right)$ & -2194.776 & -2215.322 \\
$t_{1}\left(\mathrm{MeV} \mathrm{fm}^{5}\right)$ & 482.518 & 532.536 \\
$t_{2}\left(\mathrm{MeV} \mathrm{fm}^{4}\right)$ & 138.137 & 67.761 \\
$t_{3}\left(\mathrm{MeV} \mathrm{fm}^{3(1+\sigma)}\right)$ & 10784.169 & 10931.718 \\
$x_{0}$ & 0.134 & 0.463 \\
$x_{1}$ & -0.097 & 0.128 \\
$x_{2}$ & -1.399 & -2.174 \\
$x_{3}\left(\mathrm{MeV} \mathrm{fm}^{5}\right)$ & 0.171 & 0.615 \\
$W_{0}$ & 105.674 & 116.789 \\
$\sigma$ & $1 / 6$ & $1 / 6$ \\
\hline \hline
\end{tabular}


TABLE III. Bulk nuclear matter properties obtained with the different Skyrme sets. In the last column the estimated BHF values are given. $L$ is the density slope of $a_{s}(\rho)$ times $3 \rho_{0}$.

\begin{tabular}{lccccc}
\hline \hline & LNS5 & LNS1 & LNS & SLy4 & BHF \\
\hline$\rho_{0}\left(\mathrm{fm}^{-3}\right)$ & 0.1599 & 0.1616 & 0.1746 & 0.160 & $0.16-0.17$ \\
$E / A(\mathrm{MeV})$ & -15.57 & -15.86 & -15.32 & -15.97 & $\simeq-16$ \\
$K_{\infty}(\mathrm{MeV})$ & 240.06 & 244.18 & 210.85 & 229.90 & $\simeq 220.00$ \\
$a_{s}(\mathrm{MeV})$ & 29.21 & 29.91 & 33.41 & 32.00 & $\simeq 30$ \\
$L(\mathrm{MeV})$ & 50.17 & 30.94 & 61.45 & 45.96 & $\simeq 58.20$ \\
$\frac{m *}{m}$ & 0.603 & 0.604 & 0.825 & 0.70 & $\simeq 0.633$ \\
\hline \hline
\end{tabular}

including in the pairing window only the states whose energies do not change significantly (i.e., by less than $1 \mathrm{MeV}$ ).

In Eq. (13) $\rho_{0}=0.16 \mathrm{fm}^{-3}$ while the $V_{0}$ parameter is determined by requiring a reasonable agreement with the experimental mean neutron gap along the whole series of isotopes under study. The values of $V_{0}$ thus obtained for LNS5, LNS1, and LNS are $820 \mathrm{MeV} \mathrm{fm}^{-3}, 760 \mathrm{MeV} \mathrm{fm}^{-3}$, and $780 \mathrm{MeV} \mathrm{fm}^{-3}$, respectively. In case of SLy4, $V_{0}=$ $680 \mathrm{MeV} \mathrm{fm}^{-3}$ was adopted as in Ref. [19].

The deviations of the calculated energies from the experimental values are depicted in Fig. 7. With SLy4 the pairing effects reduces the deviations by $2 \%$ at most, and tends to zero for the heaviest nuclei. In the case of LNS1 and LNS5 the deviation changes from positive to negative values from lighter to heavier isotopes, and this points to a stronger isospin dependence. The calculations show that the charge radii of $\mathrm{Sn}$ isotopes are not strongly affected by pairing.

Figure 8 summarizes the results for binding energies and charge radii of the $\mathrm{Sn}$ isotopes with the old LNS, the new sets, LNS1 and LNS5, and the reference set SLy4. From the figure it can be concluded that LNS1 and LNS5 represent a substantial improvement with respect to LNS.

TABLE IV. Binding energies in MeV (first line) and the charge radii in $\mathrm{fm}$ (second line) from LNS5 and LNS1 are compared with the experimental values. Experimental energies are taken from Ref. [17] and charge radii from Ref. [18].

\begin{tabular}{lrcc}
\hline \hline & Exp & LNS5 & LNS1 \\
\hline${ }^{16} \mathrm{O}$ & 127.62 & -137.27 & -131.52 \\
& 2.73 & 2.758 & 2.745 \\
${ }^{40} \mathrm{Ca}$ & -342.05 & -354.55 & -348.00 \\
& 3.49 & 3.551 & 3.521 \\
${ }^{48} \mathrm{Ca}$ & -415.99 & -427.13 & -421.03 \\
& 3.48 & 3.485 & 3.500 \\
${ }^{56} \mathrm{Ni}$ & -483.99 & -484.97 & -482.41 \\
& 3.75 & 3.768 & 3.751 \\
${ }^{78} \mathrm{Ni}$ & -642.40 & -653.81 & -645.24 \\
& & 3.961 & 3.973 \\
${ }^{100} \mathrm{Sn}$ & -825.78 & -823.69 & -825.81 \\
& & 4.446 & 4.457 \\
${ }^{132} \mathrm{Sn}$ & -1102.90 & -1108.23 & -1104.9 \\
& & 4.694 & 4.700 \\
${ }^{208} \mathrm{~Pb}$ & -1636.44 & -1625.41 & -1633.41 \\
& 5.50 & 5.531 & 5.531 \\
\hline \hline
\end{tabular}

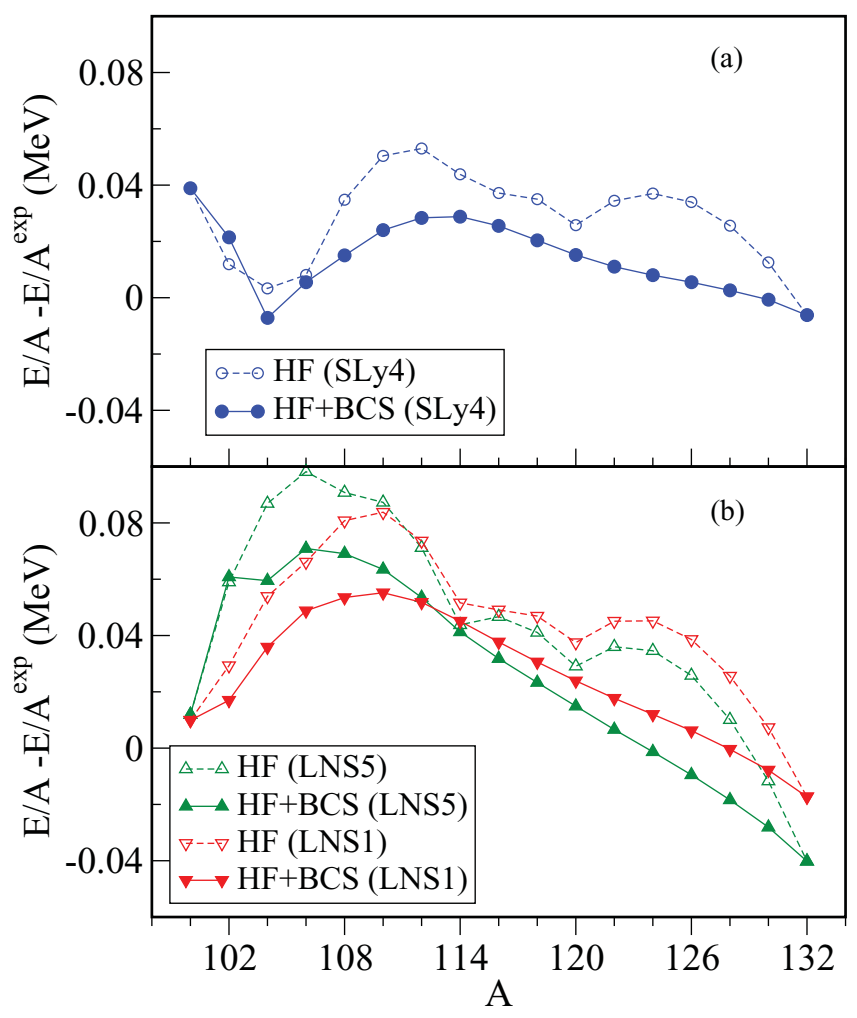

FIG. 7. (Color online) Deviations from experimental values of the Sn isotopes energies calculated with and without pairing: SLy4 parametrization (upper panel), LNS1 and LNS5 parametrizations (lower panel).

\section{B. Self-consistent RPA calculations of the giant resonance energies in ${ }^{208} \mathrm{~Pb}$}

The ground-state properties such as those discussed above play a primary role to assess the quality and the predictive power of a Skyrme force (or, more generally, of any EDF). But the properties of the excited states in finite nuclei, to which often less attention is paid, should be also considered. In particular, there exist states, like the giant resonances, whose properties carry general and relevant nuclear structure information. The random phase approximation (RPA) is closely linked to the HF mean field [20] and it is therefore appropriate to apply the RPA to cases where pairing effects are absent, such as in doubly-closed shell nuclei. In what follows, we discuss the predictions for some giant resonance states in ${ }^{208} \mathrm{~Pb}$, obtained by means of fully-self consistent HF plus random phase approximation (RPA) calculations that have been done by employing the Skyrme sets introduced in the present work.

RPA is certainly a suitable theory to describe the nuclear giant resonances (although it cannot account for their spreading width). In self-consistent RPA, the residual interaction is derived as the functional derivative (with respect to the density) of the ground-state mean field. This, in turn, is the functional derivative of the total energy. Therefore, the RPA results depend only on the parameters of the effective Skyrme Hamiltonian. 

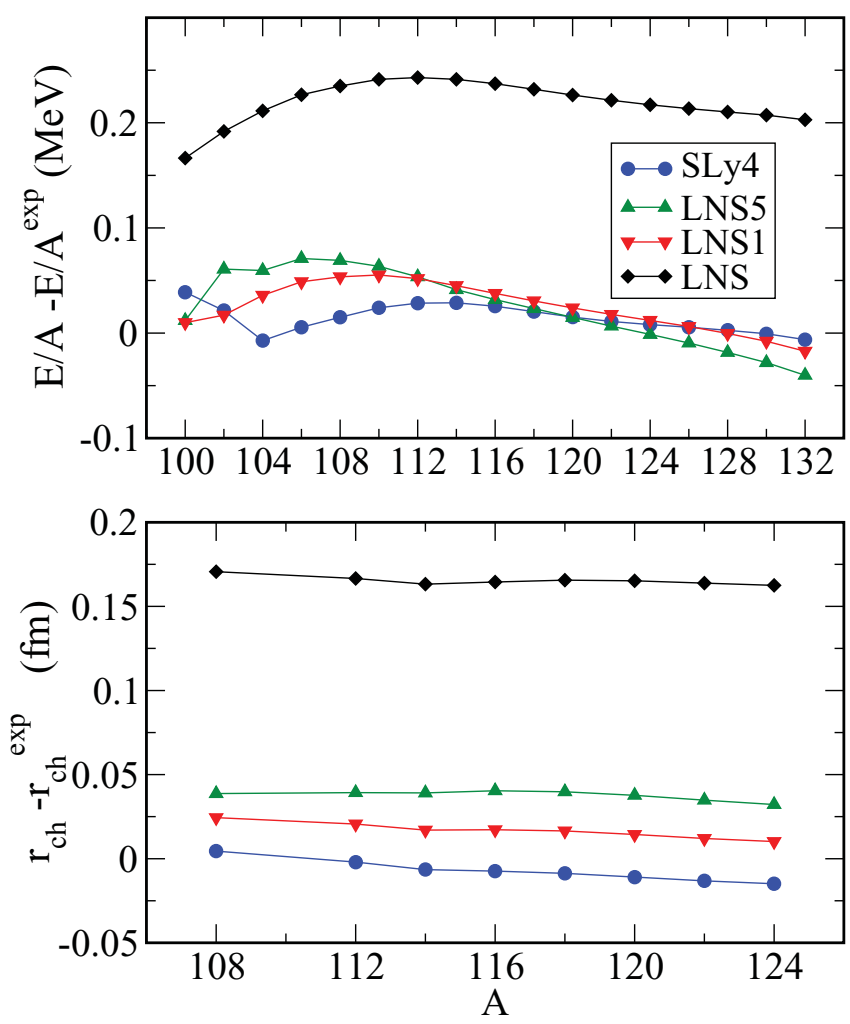

FIG. 8. (Color online) Energy (upper) and charge radii (lower) deviations with pairing correction from LNS,LNS1 and LNS5.

In the case of the RPA equations on a HF basis, the general theory is well known from textbooks [20]. In our spherical implementation (see also Refs. [21,22]), we first solve the HF equations in the coordinate space to construct the single-particle HF basis. In the present case all the radial quantities are computed up to a maximum radius of $24 \mathrm{fm}$ for ${ }^{208} \mathrm{~Pb}$, using a mesh of $0.15 \mathrm{fm}$. The unoccupied single-particle states, including those at positive energies, are obtained by putting the system in a spherical box of $24 \mathrm{fm}$ radius, i.e., the continuum is discretized. A basis of one particle-one hole $(1 p-1 h)$ configurations is then built using all occupied states, as well as the lowest unoccupied states with increasing values of the principal quantum number $n$, for each allowed value of $(l, j)$. The RPA matrix equations are then solved in this basis, which has been checked to be large enough to ensure that the appropriate energy-weighted sum rules are satisfied up to about $97-99 \%$. We should also mention that in our scheme there is no approximation in the residual interaction, in that all terms are taken into account, including those coming from the two-body Coulomb and spin-orbit forces.

After solving the RPA equations, we obtain the full set of eigenvalues and eigenvectors and from them the strength function $S(E)$ associated with a given operator $\hat{F}$. The moments of the strength function are defined as $m_{k}=\int d E S(E) E^{k}$. The centroid energy can be defined as $E_{0}=m_{1} / m_{0}$ or $E_{-1}=$ $\sqrt{m_{1} / m_{-1}}$ (more precisely, the former energy is the centroid energy whereas for the latter the name of constrained energy should be preferred).

In the case of the isoscalar monopole strength, the operator reads

$$
\hat{F}=\sum_{i=1}^{A} r_{i}^{2},
$$

where the sum runs over all nucleons. The strength function is dominated by a main peak, namely the isoscalar giant monopole resonance (ISGMR), so that centroid energy and constrained energy are quite similar with each other. In Fig. 9 we report the value of $E_{-1}$, that can be compared with the experimental finding of $14.17 \mathrm{MeV}$ [23]. The fact that our results agree with experiment can be viewed as a consequence of the nuclear incompressibility values associated with our forces, $K_{\infty}=220 \mathrm{MeV}$, a range indicated as the preferable one in Refs. [24,25]. The correlation between the ISGMR and the nuclear incompressibility had been first pointed out in Ref. [26].

The isovector giant dipole resonance (IVGDR) is the collective motion of neutrons against protons and carries information about the symmetry energy. Relationships between the dipole and the symmetry energy have been discussed in Refs. [28-30]. The associated operator, with the subtraction of the center of mass, is

$$
\hat{F}=\frac{Z}{A} \sum_{n=1}^{N} r_{n} Y_{1 M}\left(\hat{r}_{n}\right)-\frac{N}{A} \sum_{p=1}^{Z} r_{p} Y_{1 M}\left(\hat{r}_{p}\right),
$$

where the first (second) sum runs over neutrons (protons). The value of the centroid energy is shown in Fig. 9, in comparison with the experimental finding. The new parametrizations LNS1 and LNS5 slightly overestimate the data, probably not because of any drawback related with the symmetry energy, but rather owing to the low value of the effective mass. This interpretation is reinforced by the opposite result obtained with LNS, for which $m^{*} / m$ is about 0.82 . Looking at the $L$ values in Table III

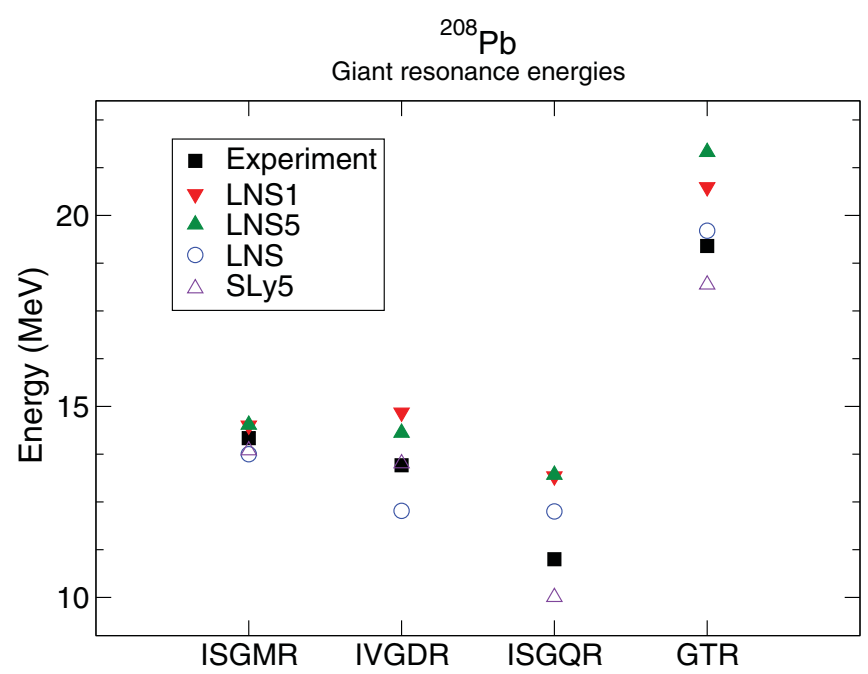

FIG. 9. (Color online) GR energies in ${ }^{208} \mathrm{~Pb} .: E_{-1}$ for monopole, centroid energies for dipole and quadrupole, and peak values for Gamow-Teller. Notice that LNS1 and LNS5 values overlap for GMR and GQR, whereas SLy5 and experimental values overlap for GMR and GDR. 
and $L=48.27 \mathrm{MeV}$ for SLy5, one may observe a correlation between the IVGDR energy and the density slope of the symmetry energy, but this cannot be easily disentangled by the effective mass. A correlation with the symmetry energy at density $0.1 \mathrm{fm}-3$ should be expected [30].

In the case of the isoscalar giant quadrupole resonance (ISGQR) it was first noticed [26,27] that the resonance energy is especially correlated with the square root of the effective mass value, that is, $\sqrt{m / m^{*}}$. The ISGQR operator is

$$
\hat{F}=\sum_{i=1}^{A} r_{i}^{2} Y_{2 M}(\hat{r}) .
$$

In Fig. 9 we show, for this operator either, the centroid energy. The fact that theory overestimates the experimental result should be once more attributed to the low effective mass.

Finally, in addition to operators that correspond only to density modes, it is useful to consider the excitation of other degrees of freedom. Spin-isospin modes have been known for a long time to have relevant collectivity. This is true, in particular, in the case of the Gamow-Teller resonance (GTR), characterized by the excitation operator

$$
\hat{F}=\sum_{i=1}^{A} \vec{\sigma}(i) \tau_{-}(i) .
$$

The centroid energy is shown in Fig. 9. In this case the LNS prediction is better than the new parametrizations and also better than SLy5. This unexpected result demands for the additional investigation on the Skyrme-type fitting of the BHF particle-hole residual interaction.

\section{CONCLUSION}

The Skyrme forces represent one of the simplest and more efficient ways to realize EDF which are alternatives to highly demanding (or prohibitive) many-body calculations based on the bare nucleon-nucleon interactions. But it is desirable the Skyrme parameters to be modeled not only on the properties of stable nuclei, but also on the properties of nuclear matter that are derived from realistic interactions. In the present work we have reexamined the LNS model of Ref. [5] and improved it in two respects. First, we use for our new set of pseudodata the results of Ref. [7] where the BHF calculations with two-body and three-body effects have been carried throughout using the parameters of the Bonn-B potential. These BHF results give a somewhat better description of the nuclear matter properties around the saturation point than the BHF results used in determining the former LNS model. Second, we have adopted a new strategy where the main quantities which determine the new LNS model are the BHF potential energies of symmetric nuclear matter in the four $(T, S)$ channels. These BHF energies are supplemented by additional constraints on binding energies and radii of some selected nuclei.

Then, new parameter sets for EDF of the Skyrme type can be obtained from least-squares fits. In this work we present as examples two sets of Skyrme parameters, LNS1 and LNS5, which improve considerably the description of binding energies and charge radii of stable nuclei as compared to the former LNS model. They have been tested in the $\mathrm{Sn}$ isotopic chain and in self-consistent RPA calculations of $J^{\pi}=0^{+}, 1^{-}, 2^{+}$giant resonances as well as GamowTeller resonances. The monopole energies are satisfactorily predicted, but a tendency to overestimate the isovector dipole and isoscalar quadrupole resonances seems to indicate that the predicted non-locality from the effective mass is too low.

In conclusion, it has been shown that BHF calculations using realistic meson exchange potentials and including three-body effects can provide a sound framework for determining EDF of the Skyrme type. The reason why the latter are not yet fully competitive with the purely phenomenological Skyrme forces is still an open problem. Nevertheless the parametrizations thus obtained can describe nuclear ground states at a reasonably good quantitative level. They might be used with more confidence than purely phenomenological parametrizations for exploring regions of the nuclear chart far from the stability valley. Finally, we can say that further improvements of microscopically based parametrizations of the Skyrme type must await for more accurate descriptions of the nuclear matter EoS in the density domain below $(0.4-0.5) \rho_{0}$. Indeed, this range of densities is important for finite nuclear systems but its treatment is generally difficult in microscopic calculations of nuclear matter.

\section{ACKNOWLEDGMENTS}

Two of the authors (D.G. and U.L.) would like to acknowledge the warm hospitality received during their visit at the Institute of Modern Physics (CAS) in Lanzhou. This work is supported in part by the Chinese Academy of Sciences visiting grant for senior international scientists, Grant no. 2009j2-26, and in part by the Italian Research Project "Many-body theory of nuclear systems and implications on the physics of neutron stars" (PRIN 2008). One of us (W.Z.) was supported by the National Natural Science Foundation of China (10875151, 10740420550), the Major State Basic Research Developing Program of China under No. 2007CB815004, the Knowledge Innovation Project (KJCX2-EW-N01) of Chinese Academy of Sciences, CAS/SAFEA International Partnership Program for Creative Research Teams (CXTD-J2005-1).

\section{APPENDIX: POTENTIAL ENERGIES PER PARTICLE IN TERMS OF SKYRME INTERACTION PARAMETERS}

The standard Skyrme interaction (without the spin-orbit and tensor components, which play no role in homogeneous matter) is

$$
\begin{aligned}
v= & t_{0}\left(1+x_{0} P_{\sigma}\right) \delta(\boldsymbol{r}) \\
& +\frac{1}{2} t_{1}\left(1+x_{1} P_{\sigma}\right)\left[\boldsymbol{k}^{\prime 2} \delta(\boldsymbol{r})+\delta(\boldsymbol{r}) \boldsymbol{k}^{2}\right] \\
& +t_{2}\left(1+x_{2} P_{\sigma}\right) \boldsymbol{k}^{\prime} \cdot \delta(\boldsymbol{r}) \boldsymbol{k} \\
& +\frac{1}{6} t_{3}\left(1+x_{3} P_{\sigma}\right) \rho(\boldsymbol{R})^{\sigma} \delta(\boldsymbol{r}),
\end{aligned}
$$

where $P_{\sigma}$ is the spin exchange operator, $\boldsymbol{k}=\left(\nabla_{1}-\nabla_{2}\right) / 2 i$ acting to the right, and $\boldsymbol{k}^{\prime}$ is its adjoint.

The different spin-isospin components $U_{T S}^{(\text {Skyrme) }}$ of the nuclear matter energy per particle calculated by using the 
Skyrme force are given by

$$
\begin{aligned}
U_{00}^{(\text {Skyrme })}(\rho)= & \frac{3}{160}\left(\frac{3 \pi^{2}}{2}\right)^{\frac{2}{3}} t_{2}\left(1-x_{2}\right) \rho^{\frac{5}{3}} \\
U_{11}^{(\text {Skyrme })}(\rho)= & \frac{27}{160}\left(\frac{3 \pi^{2}}{2}\right)^{\frac{2}{3}} t_{2}\left(1+x_{2}\right) \rho^{\frac{5}{3}} \\
\left.U_{10}^{(\text {Skyrme })} \rho\right)= & \frac{3}{16} t_{0}\left(1-x_{0}\right) \rho+\frac{9}{160}\left(\frac{3 \pi^{2}}{2}\right)^{\frac{2}{3}} t_{1}\left(1-x_{1}\right) \rho^{\frac{5}{3}} \\
& +\frac{1}{32} t_{3}\left(1-x_{3}\right) \rho^{\sigma+1}
\end{aligned}
$$

$$
\begin{aligned}
U_{01}^{(\text {Skyrme })}(\rho)= & \frac{3}{16} t_{0}\left(1+x_{0}\right) \rho+\frac{9}{160}\left(\frac{3 \pi^{2}}{2}\right)^{\frac{2}{3}} t_{1}\left(1+x_{1}\right) \rho^{\frac{5}{3}}, \\
& +\frac{1}{32} t_{3}\left(1+x_{3}\right) \rho^{\sigma+1} .
\end{aligned}
$$

The symmetry energy $E_{s}(\rho)$ and the incompressibility $K(\rho)$ of symmetric nuclear matter are

$$
\begin{aligned}
E_{s}(\rho)= & \frac{1}{3} \frac{\hbar^{2}}{2 m}\left(\frac{3 \pi^{2}}{2}\right)^{\frac{2}{3}} \rho^{\frac{2}{3}}-\frac{1}{16}\left(3 T_{0}^{+}-T_{0}^{-}\right) \rho \\
& -\frac{1}{48}\left(\frac{3 \pi^{2}}{2}\right)^{\frac{2}{3}} \rho^{\frac{2}{3}}\left(3 T_{1}^{+}-3 T_{1}^{-}-9 T_{2}^{+}+T_{2}^{-}\right) \rho^{\frac{5}{3}} \\
& -\frac{1}{96}\left(3 T_{3}^{+}-T_{3}^{-}\right) \rho^{\sigma+1}, \\
K(\rho)= & -\frac{3 \hbar^{2}}{5 m}\left(\frac{3 \pi^{2}}{2}\right)^{\frac{2}{3}} \rho^{\frac{2}{3}}+\frac{9}{32}\left(T_{3}^{+}+T_{3}^{-}\right) \sigma(\sigma+1) \rho^{\sigma+1} \\
& +\frac{3}{16}\left(\frac{3 \pi^{2}}{2}\right)^{\frac{2}{3}} \rho^{\frac{2}{3}}\left(3 T_{1}^{+}+3 T_{1}^{-}+9 T_{2}^{+}+T_{2}^{-}\right) \rho^{\frac{5}{3}},
\end{aligned}
$$

where $T_{i}^{ \pm}=t_{i}\left(1 \pm x_{i}\right)$.
[1] A. Akmal, V. R. Pandharipande, and D. G. Ravenhall, Phys. Rev. C 58, 1804 (1998).

[2] E. Chabanat, P. Bonche, P. Haensel, J. Meyer, and R. Schaeffer, Nucl. Phys. A 327, 710 (1997); 635, 231 (1998); 643, 441(E) (1998).

[3] W. Zuo, A. Lejeune, U. Lombardo, and J.-F. Mathiot, Nucl. Phys. A 706, 418 (2002).

[4] M. Baldo, C. Maieron, P. Schuck, and X. Viñas, Nucl. Phys. A 736, 241 (2004).

[5] L. G. Cao, U. Lombardo, C. W. Shen, and N. V. Giai, Phys. Rev. C 73, 014313 (2006).

[6] M. Baldo, L. Robledo, P. Schuck, and X. Viñas, J. Phys. G: Nucl. Part. Phys. 37, 064015 (2010).

[7] Z. H. Li, U. Lombardo, H. J. Schulze, and W. Zuo, Phys. Rev. C 77, 034316 (2008).

[8] A. Lejeune, U. Lombardo, and W. Zuo, Phys. Lett. B 477, 45 (2000).

[9] P. Grange, A. Lejeune, M. Martzolff, and J.-F. Mathiot, Phys. Rev. C 40, 1040 (1989).

[10] R. Machleidt, K. Holinde, and Ch. Elster, Phys. Rep. 149, 1 (1987); R. Machleidt, Adv. Nucl. Phys. 19, 189 (1989).

[11] Z. H. Li and H. J. Schulze, Phys. Rev. C 78, 028801 (2008).

[12] I. Bombaci and U. Lombardo, Phys. Rev. C 44, 1892 (1991).

[13] W. Zuo, C. W. Shen, and U. Lombardo, Phys. Rev. C 67, 037301 (2003); C. W. Shen, U. Lombardo, N. Van Giai, and W. Zuo, Phys. Rev. C 68, 055802 (2003).

[14] J. Bartel, Ph. Quentin, M. Brack, C. Guet, and H.-B. Håkansson, Nucl. Phys. A 386, 79 (1982).

[15] N. Van Giai and H. Sagawa, Phys. Lett. B 106, 379 (1981).
[16] J. Dobaczewski, H. Flocard, and J. Treiner, Nucl. Phys. A 422, 103 (1984).

[17] G. Audi and A. H. Wapstra, Nucl. Phys. A 595, 409 (1995).

[18] E. W. Otten, in Treatise on Heavy Ion Science, edited by D. A. Bromley (Plenum, New York, 1989).

[19] S. Fracasso and G. Colò, Phys. Rev. C 72, 064310 (2005).

[20] D. J. Rowe, Nuclear Collective Motion (Methuen, London, 1970); P. Ring and P. Schuck, The Nuclear Many-Body Problem (Springer-Verlag, Berlin, 1980).

[21] G. Colò, P. F. Bortignon, S. Fracasso, and N. Van Giai, Nucl. Phys. A 788, 173c (2007).

[22] G. Colò, L. Cao, N. Van Giai, and L. Capelli, Comput. Phys. Commun. (submitted).

[23] D. H. Youngblood, H. L. Clark, and Y. W. Lui, Phys. Rev. Lett. 82, 691 (1999).

[24] G. Colò, N. V. Giai, J. Meyer, K. Bennaceur, and P. Bonche, Phys. Rev. C 70, 024307 (2004).

[25] S. Shlomo, V. M. Kolomietz, and G. Colò, Eur. Phys. J. A 30, 23 (2006).

[26] J. P. Blaizot, Phys. Rep. 64, 171 (1980).

[27] O. Bohigas, A. M. Lane, and J. Martorell, Phys. Rep. 51, 267 (1979).

[28] A. W. Steiner, M. Prakash, J. M. Lattimer, and P. J. Ellis, Phys. Rep. 411, 325 (2005).

[29] G. Colò, N. Van Giai, and H. Sagawa, Phys. Lett. B 363, 5 (1995).

[30] L. Trippa, G. Colò, and E. Vigezzi, Phys. Rev. C 77, 061304(R) (2008). 